\title{
Uncertainties in Estimating $B_{c}$ Hadronic Production and Comparisons of the Production at TEVATRON and LHC
}

\author{
Chao-Hsi Chang ${ }^{1,2} *$ and Xing-Gang $\mathrm{Wu}^{2,3 \dagger}$ \\ ${ }^{1}$ CCAST (World Laboratory), P.O.Box 8730, Beijing 100080, China. ${ }^{\ddagger}$ \\ ${ }^{2}$ Institute of Theoretical Physics, Chinese Academy of Sciences, \\ P.O.Box 2735, Beijing 100080, China. \\ ${ }^{3}$ Institute of High Energy Physics, P.O.Box 918(4), Beijing 100039, China
}

\begin{abstract}
The uncertainties in estimating the hadronic production of the $B_{c}$ meson are studied under the framework of the complete $\alpha_{s}^{4}$ approach of the perturbative QCD and the gluon-gluon fusion mechanism. Quantitative comparisons on the production at TEVATRON and LHC are made. Considering the detectors at TEVATRON and LHC, we have also estimated the production with proper kinematic cuts. Based on the results, we conclude that the experimental studies of the $B_{c}$ meson at the two colliders will be complimentary and stimulative. We find that as c.m. energy is increasing from RUN-I to RUN-II at TEVATRON, the production cross-section increases about $20 \%$.
\end{abstract}

PACS numbers: 12.38.Bx, 13.85.Ni, 14.40.Nd, 14.40.Lb.

Keywords: $B_{c}$ meson, inclusive hadronic production, uncertainties.

\footnotetext{
* Email: zhangzx@itp.ac.cn

$\dagger$ Email: wuxg@mail.ihep.ac.cn

$\ddagger$ Not correspondence address.
} 


\section{INTRODUCTION}

$B_{c^{-}}$-physics is attracting more and wide interests due to experimental progresses 1, 2, 3] and theoretical ones [4, 5, 6, 7, 8, 9, 10, 11, 12, 13, 14, 15, 16, 17, 18, 19, 20, 21, 22] (especially the experimental discovery 3]). Since the production cross section is relatively small in comparison with the production of the other heavy mesons ( $B, D$ etc) and heavy quarkonia $(J / \psi, \Upsilon$ etc), only at high energy hadronic colliders with high luminosity, can one collect enough $B_{c}$ events for experimental studies [4, 5, 6, 7, 8, 9, 10, 11]. Considering the potential applications to the experimental feasibility studies on the topic, a computer program for hadronic generating the $B_{c}$ meson, BCVEGPY, has been completed in Ref. 23], which has been written in Fortran and can be conveniently implemented into PYTHIA 24].

The hadronic production of the $B_{c}$-states with different spin has been estimated by a lot of authors, and all are mainly based on the gluon-gluon fusion mechanism, i.e. taking the hard subprocess $g g \rightarrow B_{c}+b+\bar{c}$ as dominant one. The authors of [9, 12] gave the estimate in terms of the 'fragmentation approach', and the authors of [8, 10, 11] did it in the so-called 'complete approach'. The results of the two approaches agree essentially within theoretical uncertainties, especially, at high $B_{c}$ transverse momentum $P_{T}$ [10] and when the fragmentation approach additionally takes into account the contributions from gluon fragmentation [12]. The fragmentation approach is appropriate if one is only interested in the information of the produced $B_{c}$ itself, and its accuracy is improving with the increasing of $P_{T}$ [10]. Comparatively, the advantage of the complete approach is that it can also retain the information of the accompanied $\bar{c}$ and $b$ quarks (jets). For experiments, to retain more information of the events is more relevant. Therefore, in BCVEGPY [23], the complete calculation approach has been adopted.

Moreover according to perturbative QCD (pQCD), in addition to the gluon-gluon fusion mechanism, there are several different mechanisms for the production, such as that via the quark-antiquark annihilation subprocess $q \bar{q} \rightarrow B_{c}+b+\bar{c}$, and that via the color octet mechanism and etc.. In Ref. [8], it is shown that the contributions to the production from quark-antiquark annihilation are much smaller than those from gluon-gluon fusion. It is mainly because of the fact that the relevant luminosity for gluon-gluon fusion is a product of the parton distribution functions (PDFs) for gluon components in the colliding hadrons, whereas 6that for quark-antiquark annihilation is a product of the quark and anti-quark 
distribution functions respectively while in a high energy collider, such as TEVATRON $(p \bar{p}$ collision $\sqrt{S}=2 \mathrm{TeV})$ at Fermilab and LHC $(p p$ collision $\sqrt{S}=14 \mathrm{TeV})$ at CERN, the luminosity for gluon-gluon fusion due to parton distribution functions (PDFs) at the most kinematic region is much higher than that for quark-antiquark annihilation [27]. Furthermore, in the annihilation subprocess, there is an additional $S$-channel suppression from the virtual gluon propagator, which will also make contributions to the production cross section small. In the subsection II-C we will make a brief comparison between those two mechanisms. According to non-relativistic QCD (NRQCD) 25], for a double heavy meson, such as $J / \psi, \eta_{c}, B_{c}, \cdots$, there may be additional color-octet components which may contribute the production substantially. The magnitude of the color-octet components may be estimated with the NRQCD velocity scaling rule $\left(v \simeq \alpha_{s}\left(m_{B_{c}}\right) \sim 0.1\right)$. One may find that there is no enhancement in the hadronic production (in contract to of the hidden flavored double heavy objects such as $J / \psi, \psi^{\prime}$ the production which can gain one order or more in $\alpha_{s}$ via the color octet components) and the color octet components in $B_{c}$ are definitely smaller than the color singlet ones (with the same input parameters, the total contributions to the hadronic production is $\lesssim v \sim v^{2}$ of the color-singlet one), therefore, the contributions from color octet components of $B_{c}$ are ignorable at leading order.

The contributions to the production from the color octet and those from the quarkantiquark annihilation mechanism were neglected in Refs. [9, 10, 11, 12, 13]. In BCVEGPY 23] and thus in the present paper, the mechanisms via color-octet for the $B_{c}$ production are ignored.

Although the estimated values for the hadronic production of $B_{c}$ in literature $9,10,11$, 12] are consist within the theoretical uncertainties, in a few cases the differences on the production can reach up to almost one magnitude order. Moreover, RUN-II of TEVATRON is taking data, LHC is under constructing, and various experimental feasibility studies of $B_{c}$ are in progress, thus we think to know the theoretical uncertainties quantitatively in estimates of the $B_{c}$ production, in addition, the precise comparisons of the production at LHC and TEVATRON are interesting.

The newly developed generator BCVEGPY has been well tested by carefully comparing the results with those in earlier references [8]. It, having been implemented into PYTHIA, can be conveniently applied to simulate $B_{c}$ events (Monte Carlo simulation). In the paper, we highlight the uncertainties of the dominant gluon-gluon fusion mechanism, make a 
brief comparison on the gluon-gluon fusion mechanism and the quark-antiquark annihilation mechanism but precise comparisons on the production at TEVATRON and LHC. Although some certain uncertainties will be able to understand (control) better when the next to leading order (NLO) pQCD calculation is achieved, considering that the NLO calculation cannot be available soon due to its complicatedness, we restrict ourselves here to examine the uncertainties only 'up to' the lowest order. The uncertainties that we will examine in the paper, include the variations about $\alpha_{s}$-running, the choices of the factorization energy scale, the various versions of parton distribution functions (PDFs), the values of the bound state parameters and etc.. To be useful references, the production with possible kinematic cuts, which roughly 'match' the detectors at TEVATRON and at LHC, is also investigated.

It is known that at high energy hadronic colliders, numerous $B_{c}$ events may be produced, moreover due to the fact that the $B_{c}$ meson has a quite large branching ratio to decay into a $B_{s}$ meson (several tens percent) 16, 17, 18], thus, in principle, copious and precisely tagged $B_{s}$ mesons at production position may be collected through the inclusive decays of $B_{c}$ at the hadronic colliders $\left(B_{c}\left(\bar{B}_{c}\right)\right.$ is the charged meson, as long as the charge of $B_{c}\left(\bar{B}_{c}\right)$ and/or its decay products is determined, the produced $B_{s}\left(\bar{B}_{s}\right)$ mesons through the decays of $B_{c}$ $\left(\bar{B}_{c}\right)$ are tagged precisely at the decay vertex of $\left.B_{c}\left(\bar{B}_{c}\right)\right)$. To study $B_{s}-\bar{B}_{s}$ mixing and $C P$ violation are very interesting topics and can be done only in hadronic environment. For experimental studies of $B_{s}-\bar{B}_{S}$ mixing and certain $C P$ violation in $B_{s}$ decays, the $B_{s}$ mesons being tagged at their production position are crucial. In hadronic environment to obtain copious $B_{s}$ mesons which are precisely tagged at their production positions, the way through $B_{c}$ decays is worth for considering seriously especially at LHC. In addition, potentially this way has a great advantage in rejecting backgrounds and deducing the systematic errors [5]. Therefore the feasibility for studying the $B_{s}$ mesons in this way should be investigated carefully, especially, by means of Monte Carlo simulating the events under specific detector environment so as to see the precise efficiency to have the tagged $B_{s}\left(B_{s}\right)$ mesons and whether the idea is really practicable or not.

The paper is organized: following Introduction, in Section II we present the studies of the uncertainties in the estimates of the hadronic production for the dominant mechanism of gluon-gluon fusion and also a brief comparison between the mechanisms of gluon-gluon fusion and quark-antiquark annihilation. In Section III, we present the production with possible kinematic cuts, which roughly match the detector situation at TEVATRON and 

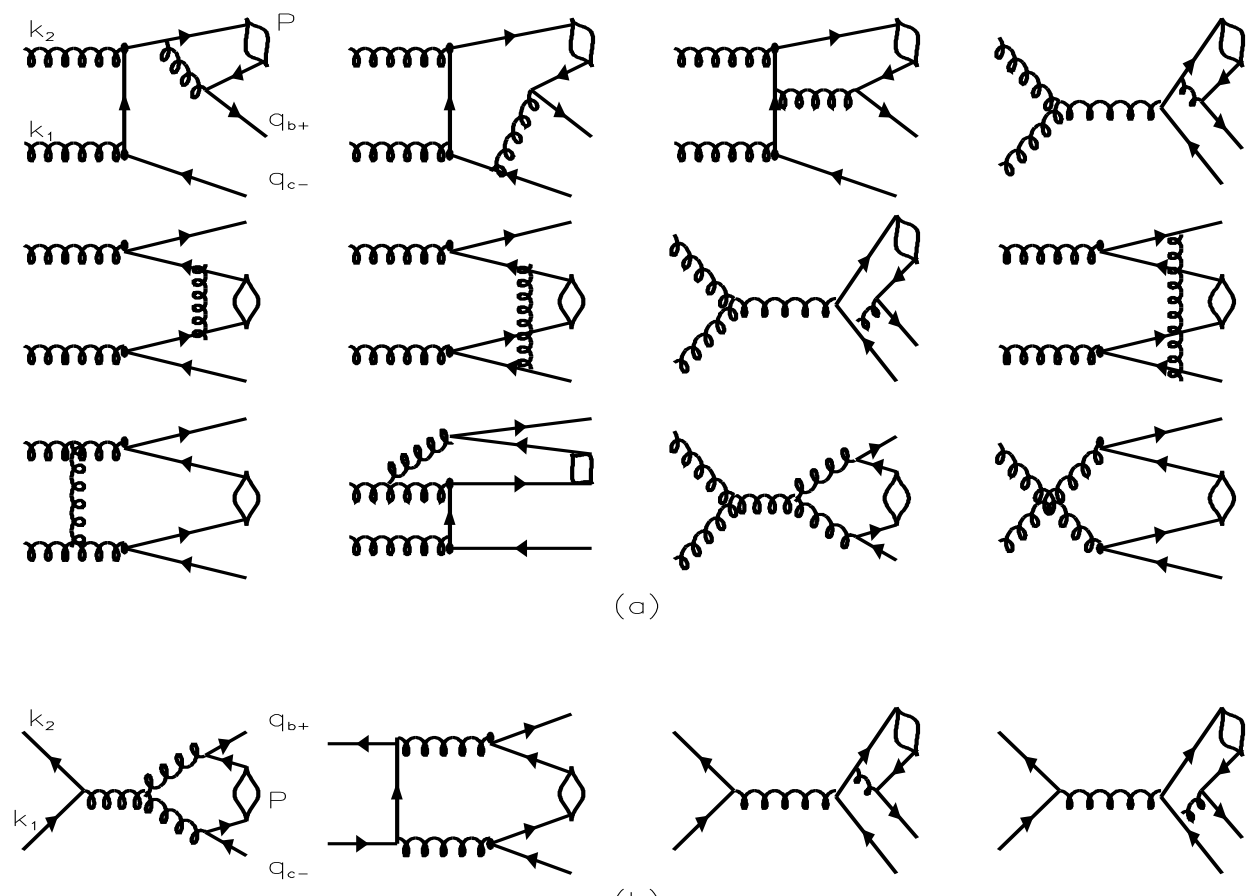

FIG. 1: (a). The typical Feynman diagrams for the gluon-gluon fusion subprocess; (b). The typical Feynman diagrams for quark-antiquark annihilation subprocess.

LHC. We also compute the production at TEVATRON with C.M. energy changes from RUN-I to RUN-II. In the last section, we make some discussions and a short summary.

\section{THE UNCERTAINTIES IN ESTIMATES}

As stated in the introduction, there are two mechanisms for the lowest order of pQCD $\alpha_{s}^{4}$ approach in the hadronic $B_{c}$ production, the gluon-gluon fusion and the quark-antiquark annihilation. Of them the gluon-gluon fusion mechanism is the dominant one, and there are 36 Feynman diagrams for its subprocess. The typical Feynman diagrams for the subprocess are plotted in FIG.1(a). In comparison, for the other mechanism, its subprocess is of quarkantiquark annihilation and there are 7 Feynman diagrams. The typical ones are plotted in FIG.1(b). In fact, if we consider the production only through the dominant gluon-gluon fusion mechanism, then the contributions from the quark-antiquark annihilation mechanism will be considered as one kind of uncertainties. In this section, firstly we will focus on the gluon-gluon fusion mechanism and then we will make a brief comparison between these two mechanisms. 
To be more useful experimentally, we study the uncertainties of $B_{c}$ hadronic production by means of the 'complete approach', i.e., at collision C.M. energy $\sqrt{S}$,

$$
\begin{gathered}
d \sigma\left(S, p_{T}, \cdots\right)=\sum_{i j} \int d x_{1} \int d x_{2} F_{H_{1}, P_{1}}^{i}\left(x_{1}, \mu_{F}^{2}\right) \cdot F_{H_{2}, P_{2}}^{j}\left(x_{2}, \mu_{F}^{2}\right) \\
\cdot d \hat{\sigma}_{i j \rightarrow B_{c} b \bar{c}}\left(P_{1}, P_{2}, x_{1}, x_{2}, \mu_{F}^{2}, \hat{s}, p_{T}, \cdots\right)
\end{gathered}
$$

where $F_{H_{1}, P_{1}}^{i}\left(x_{1}, \mu_{F}^{2}\right)$ and $F_{H_{2}, P_{2}}^{j}\left(x_{2}, \mu_{F}^{2}\right)$ are the PDFs of incoming hadrons $H_{1}$ (momentum $P_{1}$ ) and $H_{2}$ (momentum $P_{2}$ ) for parton $i$ (with the momentum fraction $x_{1}$ ) and parton $j$ (with the momentum fraction $x_{2}$ ) respectively; $\mu_{F}$ is the energy scale where the factorization and renormalization for the PDFs and the hard subprocess are made; $d \hat{\sigma}_{i j \rightarrow B_{c} b \bar{c}}$ is the differential cross-section of the relevant hard subprocess, in which $\hat{s}=x_{1} x_{2} S$ is the c.m.s. energy of the subprocess and $p_{T}$ is the transverse momentum of $B_{c}$. In the sub-sections below, we discuss the uncertainties which are from the non-perturbative factors and from those related to the hard subprocess.

\section{A. The Uncertainties Relevant to the Parameters of Potential Model and Masses} of Quarks and the Meson

For all the approaches and the color singlet mechanisms to the $B_{c}$ production, the 'decay constant' $f_{B_{c}}$ relating the hadron matrix element, as one of important input-parameters, is needed as input, and so far it may be calculated either by potential model [14] or by lattice QCD [15], while in the parer, we will make discussions related to it on potential model only. Within potential model, it directly relates to the wave function at the origin of the binding system in terms of the following formula,

$$
f_{B_{c}}^{2}=\frac{12\left|\Psi_{[1 S]}(0)\right|^{2}}{m_{B_{c}}},
$$

where $m_{B_{c}}$ is the $B_{c}$ meson mass, $\Psi_{[1 S]}$ is the wave function at the origin of the binding system $(c \bar{b})$ at the $[1 S]$ level. Since the spin splitting effects are ignored here, so there is no difference for the decay constant between the spin stats $\left[{ }^{1} S_{0}\right]$ and $\left[{ }^{3} S_{1}\right]$. There are several parameters in the potential model, which need to be fixed by fitting the experimental data of the heavy quarkonia $((c \bar{c})$ and $(b \bar{b}))$, so when computing the spectrum and the wave functions of the $(c \bar{b})$ system $[14]$, certain uncertainties from the fitting procedure will cause some uncertainties of $f_{B_{c}}$. In the leading order $(\mathrm{LO})$ approximation for the production, $f_{B_{c}}$ 
TABLE I: The total cross section (in unit nb) for hadronic production of $B_{c}\left[1^{1} S_{0}\right]\left(B_{c}^{*}\left[1^{3} S_{1}\right]\right)$ with various values of the $c$-quark mass $m_{c}$ and fixed $b$-quark mass $m_{b}=4.9 \mathrm{GeV}$. For definiteness and focussing the uncertainties from the quark masses alone, the rest uncertainty sources are fixed: the mass of the meson $B_{c}, m_{B_{c}}=m_{c}+m_{b}$; the gluon distribution function is taken from CTEQ5L; the factorization energy scale is chosen $\mu_{F}^{2}=Q^{2}=\hat{s} / 4$ and the running $\alpha_{s}$ is of leading order.

\begin{tabular}{|c||c|c|c|c|c|c||c|c|c|c|c|c|}
\hline \multicolumn{1}{|c||}{} & \multicolumn{4}{c||}{ TEVATRON $(\sqrt{S}=2 . \mathrm{TeV})$} & \multicolumn{5}{c|}{$\operatorname{LHC}(\sqrt{S}=14 . \mathrm{TeV})$} \\
\hline \hline$m_{c}(\mathrm{GeV})$ & 1.3 & 1.4 & 1.5 & 1.6 & 1.7 & 1.8 & 1.3 & 1.4 & 1.5 & 1.6 & 1.7 & 1.8 \\
\hline$\sigma_{B_{c}}(n b)$ & 4.84 & 3.87 & 3.12 & 2.56 & 2.12 & 1.76 & 75.6 & 61.0 & 49.8 & 41.4 & 34.7 & 28.9 \\
\hline$\sigma_{B_{c}^{*}}(n b)$ & 12.3 & 9.53 & 7.39 & 5.92 & 4.77 & 3.87 & 194. & 153. & 121. & 97.5 & 80.0 & 66.2 \\
\hline \hline
\end{tabular}

appears in the amplitude as a linear factor exactly, so the production cross sections are proportional to it squared. Therefore, the uncertainties in the production from $f_{B_{c}}$ can be figured out straightforwardly, so throughout the paper, we will fix the value $f_{B_{c}}=0.48 \mathrm{GeV}$ for the ground states (the relevant uncertainties would be figured out if need).

In addition to the decay constant $f_{B_{c}}$, the quark mass values $m_{c}$ and $m_{b}$ also 'generate' uncertainties for the hadronic production. If all of the rest parameters in the potential might be fixed, then the values of quark masses would determine the decay constant $f_{B_{c}}$, the meson mass $m_{B_{c}}$ and etc. completely. However at present, the parameters in potential model, which also appear in the formula Eq.(11), cannot be completely fixed by fitting the available data of the heavy quarkonia, so the relations of the quark masses to the decay constant $f_{B_{c}}$, the meson mass and etc. cannot be well determined. Therefore, when studying the uncertainties for the hadronic production, we can consider all of the factors, explicitly appearing in the potential model and in the formula for the subprocess Eq.(11), in a 'factorized' way. Furthermore, since $B_{c}$ is the non-relativistic and weak-binding bound state, at LO the relative momentum between the constitute quarks can be ignored, e.g., we approximately have $m_{B_{c}} \simeq m_{b}+m_{c}$.

Throughout the paper, we study the uncertainties in 'a factorization way', i.e., all of the parameters vary independently in their reasonable regions. For instance, when focussing on 
TABLE II: The total cross section (in unit nb) for hadronic production of $B_{c}\left[1^{1} S_{0}\right]$ and $B_{c}^{*}\left[1^{3} S_{1}\right]$ with various values of the $b$-quark mass $m_{b}$ and fixed $c$-quark mass $m_{c}=1.5 \mathrm{GeV}$. For definiteness and focussing the uncertainties from the quark masses alone, the gluon distribution function is taken from CTEQ5L; the factorization energy scale is chosen $\mu_{F}^{2}=Q^{2}=\hat{s} / 4$ and the running $\alpha_{s}$ is of leading order.

\begin{tabular}{|c||c|c|c|c|c||c|c|c|c|c|}
\hline \hline \multicolumn{1}{|c||}{} & \multicolumn{4}{c||}{ TEVATRON $(\sqrt{S}=2 . \mathrm{TeV})$} & \multicolumn{5}{c|}{$\operatorname{LHC}(\sqrt{S}=14 . \mathrm{TeV})$} \\
\hline \hline$m_{b}(\mathrm{GeV})$ & 4.5 & 4.7 & 4.9 & 5.1 & 5.3 & 4.5 & 4.7 & 4.9 & 5.1 & 5.3 \\
\hline$\sigma_{B_{c}}(n b)$ & 4.10 & 3.55 & 3.12 & 2.70 & 2.38 & 63.4 & 56.2 & 49.8 & 44.1 & 39.6 \\
\hline$\sigma_{B_{c}^{*}}(n b)$ & 9.43 & 8.35 & 7.39 & 6.59 & 5.89 & 148. & 133. & 121. & 110. & 100. \\
\hline \hline
\end{tabular}

the uncertainties from $m_{c}$, we let it be a basic 'input' parameter varying in a possible range

$$
1.3 \mathrm{GeV} \leq m_{c} \leq 1.8 \mathrm{GeV}
$$

with all the other factors, including the $b$-quark mass, the decay constant $f_{B_{c}}$ and etc. being fixed.

The uncertainties from $m_{c}$ are indicated by the calculated total cross sections with the $m_{c}$ of Eq.(3), which are given in TABLE[ Note that for the mass of $B_{c}$, the experimental result is $m_{B_{c}}=6.4 \pm 0.4 \mathrm{GeV}$ [3], while the prediction by the potential model gives $m_{B_{c}}=6.1 \sim 6.3$ $\mathrm{GeV}$ [14] and that by lattice QCD is about $6.4 \mathrm{GeV}$ [15]. Thus with $m_{b}=4.9 \mathrm{GeV}$ and $m_{B_{c}} \simeq m_{b}+m_{c}$, the obtained $m_{B_{c}}$ is in the region of theoretical prediction as well as experimental measurement. The uncertainties from $m_{b}$ can be analyzed in a similar way, where the $b$-quark mass runs over the range:

$$
4.5 \mathrm{GeV} \leq m_{b} \leq 5.3 \mathrm{GeV} .
$$

The results for various b-quark mass are put in TABLE【 In Tables凹and the total crosssection for the hadronic production of $B_{c}\left[1^{1} S_{0}\right]$ and $B_{c}^{*}\left[1^{3} S_{1}\right]$ at TEVATRON and LHC are computed, where the other factors are fixed precisely as: the PDFs are taking as CTEQ5L 26]; the strong coupling $\alpha_{s}$ is in leading order and the factorization energy scale is taken to be $\mu_{F}^{2}=Q^{2}=\hat{s} / 4$, where $\hat{s}$ is the C.M. energy squared of the subprocess.

From the tables, one may observe how the values of $m_{c}$ and $m_{b}$ affect the cross section up to a sizable degree. Roughly speaking, both at TEVATRON and LHC, when $m_{b}$ increases by 
steps of $0.2 \mathrm{GeV}$, then the cross section decreases by $10 \% \sim 20 \%$, while when $m_{c}$ increases by steps of $0.1 \mathrm{GeV}$, the cross section also decreases by $10 \% \sim 20 \%$.

In further investigations of the paper when examining the uncertainties from the rest factors, we shall take the values, $m_{c}=1.5 \mathrm{GeV}$ and $m_{b}=4.9 \mathrm{GeV}$, for the quark masses.

\section{B. Uncertainties Relevant to the QCD Parameters and PDFs}

In this sub-section, let us focus on the gluon-gluon fusion mechanism only.

As is shown in Eq.(11), PDFs $F_{H_{1}\left(P_{1}\right)}^{i}\left(x_{1}, \mu_{F}^{2}\right)$ and $F_{H_{2}\left(P_{2}\right)}^{j}\left(x_{2}, \mu_{F}^{2}\right)$ with $H_{1}=$ proton, $H_{2}=$ proton for LHC; $H_{1}=$ proton, $H_{2}=$ anti-proton for TEVATRON generate uncertainties in the production. PDFs are of non-perturbative nature, and in Eq.(11) they have been factorized out at the energy scale $\mu_{F}^{2}$ with the help of pQCD factorization theorem. Usually the factorization is carried out at $Q^{2}$, a characteristic energy scale for the hard subprocess, i.e. $\mu_{F}^{2}=Q^{2}$, so we use $Q^{2}$ and $\mu_{F}^{2}$ on the equal footing throughout the paper.

As stated in the Introduction, all the estimates of the production are of leading (the lowest) order of pQCD and then how to choose the energy scale $Q^{2}$ is a very tricky problem. If $Q^{2}$ is chosen properly, the results may be quite accurate[31]. From experience, for a hard subprocess with a two-body final state, generally when taking $Q^{2}=\frac{1}{4} \hat{s}(\sqrt{\hat{s}}$ is the C.M. energy of the subprocess), we can achieve quite an accurate result for LO calculations. However, in the present case, the gluon-gluon fusion subprocess is of three-body final state and contain heavy quarks, so there are ambiguities in choosing $Q^{2}$ and various choices of $Q^{2}$ would generate quite different results. Since such kind of ambiguity cannot be justified by the LO calculation itself, so we take it as the uncertainty of the LO calculation, although when the NLO calculation of the subprocess is available, the uncertainty will become under control a lot. While the NLO calculation is very complicated and it cannot be available in the foreseeable future, so here we take $Q^{2}$ as a possible characteristic momentum of the hard subprocess being squared. Having reviewed the choices of $Q^{2}$ in the literature, in the following we choose a few typical examples to study this kind of uncertainties.

According to the factorization, the running of $\alpha_{s}$ and PDFs should be of leading logarithm order (LLO), and the energy scale $Q^{2}$ appearing in the calculation should be taken as one of the possible characteristic energy scales of the hard subprocess. In principle, as has been shown in references [7, 8, 9, 10, 11, 12, 13], there are several typical ways to choose the energy 
scale $Q^{2}$ and to relate it to a characteristic energy scale $\left(Q^{2} \gg \Lambda_{Q C D}^{2}\right)$ of the subprocess, such as $Q^{2}=4 m_{c}^{2}, Q^{2}=4 m_{b}^{2}, Q^{2}=p_{T}^{2}+m_{B_{c}}^{2}$ (the 'transverse mass' squared of $B_{c}$ ), $Q^{2}=\frac{1}{4} \hat{s}$ and etc.. When studying the uncertainties on the various choices of $Q^{2}$, we precisely choose the following four types of $Q^{2}$ :

Type A: $Q^{2}=\hat{s} / 4$, the C.M. energy squared of the subprocess that is divided by 4 ;

Type B: $Q^{2}=p_{T}^{2}+m_{B_{c}}^{2}$, the transverse mass squared of the $B_{c}$ meson;

Type C: $Q^{2}=\hat{s}$, the C.M. energy squared of the subprocess;

Type $\mathrm{D}, Q^{2}=p_{T b}^{2}+m_{b}^{2}$, the transverse mass squared of the $b$ quark.

Since the PDFs can be obtained only through global fitting of the experimental data and evolute them to the requested characteristic scale $Q^{2}$ in standard way of $\mathrm{pQCD}$, so there are several groups, CTEQ [26, 27], GRV 28] and MRS [29] etc, who devote themselves to offer accurate PDFs to the world, and to keep PDFs updated as soon as possible when new relevant experimental data are available. Thus in literature, different versions of PDFs (including different issues by the same group) are used in the estimates of the hadronic production. To study the uncertainties due to various versions of PDFs, we consider the above mentioned three groups.

The generator BCVEGPY is programmed based on the dominant subprocess to LO pQCD only, to be self-consistent, when applying BCVEGPY we shall adopt the LO PDF. Thus we take CTEQ6L [27], CTEQ5L [26], GRV98L 28] and MRST2001L[29] as typical examples for PDFs. The versions of the gluon distributions ended with ' $\mathrm{L}$ ' are accurate up-to the leading logarithm order (LLO), i.e., their QCD evolution effects with $\alpha_{s}$ running are included, so for the production to show the uncertainties correctly up to LO accuracy, it is necessary for the PDFs, the hard subprocess and the QCD 'coupling constant' $\alpha_{s}$ 'run' to the energy scale $Q^{2}$ properly. The running $\alpha_{s}$ in the PDFs and in the subprocess should be the same, and then when computing the production and taking the PDFs from one version of the three groups, the running $\alpha_{s}$ should also be taken from the same group.

For comparison between TEVATRON and LHC and to pinpoint the uncertainties from PDFs, $\alpha_{s}$ running and the choices of the characteristic energy scale $Q^{2}$, we calculate the production cross sections according to four types of PDFs, the strong coupling $\alpha_{s}$ up to LLO fixed by the PDFs and the characteristic $Q^{2}$ chosen as Type A and Type B. The obtained results are shown in TABLE III. From the table, we can see that the gluon distribution functions, the running coupling constant and the corresponding energy scale, all affect the 
TABLE III: Total cross-section for the hadronic production of $B_{c}\left[1^{1} S_{0}\right]$ and $B_{c}^{*}\left[1^{3} S_{1}\right]$ at TEVATRON and at LHC with the leading order (LLO) running $\alpha_{s}$ and the characteristic energy scale $Q^{2}=\hat{s} / 4$ or $Q^{2}=p_{T}^{2}+m_{B_{c}}^{2}$. The cross section is in unit of nb.

\begin{tabular}{|c||c|c|c|c||c|c|c|c|}
\hline \hline & CTEQ5L & CTEQ6L & GRV98L & MRST2001L & CTEQ5L & CTEQ6L & GRV98L & MRST2001L \\
\hline- & \multicolumn{7}{c||}{$Q^{2}=\hat{s} / 4$} & \multicolumn{5}{c|}{$Q^{2}=p_{T}^{2}+m_{B_{c}}^{2}$} \\
\hline \hline \multicolumn{10}{||||}{} & \multicolumn{7}{|c|}{ TEVATRON } \\
\hline$\sigma_{B_{c}\left(1^{1} S_{0}\right)}$ & 3.12 & 3.79 & 3.27 & 3.40 & 4.39 & 5.50 & 4.54 & 4.86 \\
\hline$\sigma_{B_{c}^{*}\left(1^{3} S_{1}\right)}$ & 7.39 & 9.07 & 7.88 & 8.16 & 10.7 & 13.4 & 11.1 & 11.9 \\
\hline \hline- & & & \multicolumn{7}{c|}{ LHC } \\
\hline$\sigma_{B_{c}\left(1^{1} S_{0}\right)}$ & 49.8 & 53.1 & 53.9 & 47.5 & 65.3 & 71.1 & 70.0 & 61.4 \\
\hline$\sigma_{B_{c}^{*}\left(1^{3} S_{1}\right)}$ & 121. & 130. & 131. & 116. & 164. & 177. & 172. & 153. \\
\hline \hline
\end{tabular}

cross section to certain degrees.

For all of the LO PDFs, the differences in the corresponding cross sections given by them are small (about 10\% - 20\%). But the choice of $Q^{2}$ as Type A and Type B causes the cross section to change by a factor of about $\frac{1}{4}$ to $\frac{1}{3}$, which is a comparatively large effect. From TABLE III, one may see that the cross section at TEVATRON and at LHC presents a somewhat different aspect of the cross section. Roughly speaking at TEVATRON CTEQ6L is the biggest, while at LHC, GRV98L is the biggest, under a certain characteristic energy scale such as $Q^{2}$ in type A.

From TABLE [II, we may also see that the cross section of the $B_{c}$ meson production at LHC are at least one order larger in magnitude than that at TEVATRON. This is mainly due to the fact that LHC has much higher collide energy than TEVATRON, so the lowest boundary of the momentum fractions $x_{i} \quad(i=1,2)$ at LHC are much smaller than that at TEVATRON and then there are more interacting partons i.e. gluons, which have a C.M. energy above the threshold for the subprocess, in the collision hadrons at LHC than at TEVATRON.

When the produced $B_{c}$ mesons with a small $p_{T}$ are too close to the collision beam, they cannot be measured experimentally, so we calculate the cross-sections with transversemomentum cuts $p_{T c u t}$. For comparison, we define the following ratio of the integrated cross 
TABLE IV: Dependence of the ratio $R=\left(\frac{\sigma_{T E V T R O N}}{\sigma_{L H C}}\right)$ on $p_{T c u t}$ for $B_{c}\left[1^{1} S_{0}\right]$, where $Q^{2}=\hat{s} / 4$, the running $\alpha_{s}$ is in LO and the gluon distribution is CTEQ5L.

\begin{tabular}{|c||c|c|c|c||c|c|c|c|}
\hline \hline \multicolumn{1}{|c||}{} & \multicolumn{5}{c||}{$B_{c}$} & \multicolumn{4}{c|}{$B_{c}^{*}$} \\
\hline$P_{\text {Tcut }}(\mathrm{GeV})$ & 0 & 5 & 50 & $100^{‘}$ & 0 & 5 & 50 & 100 \\
\hline \hline$R\left(\times 10^{-2}\right)$ & 6.26 & 5.24 & 1.00 & 0.31 & 6.09 & 5.19 & 0.97 & 0.24 \\
\hline \hline
\end{tabular}
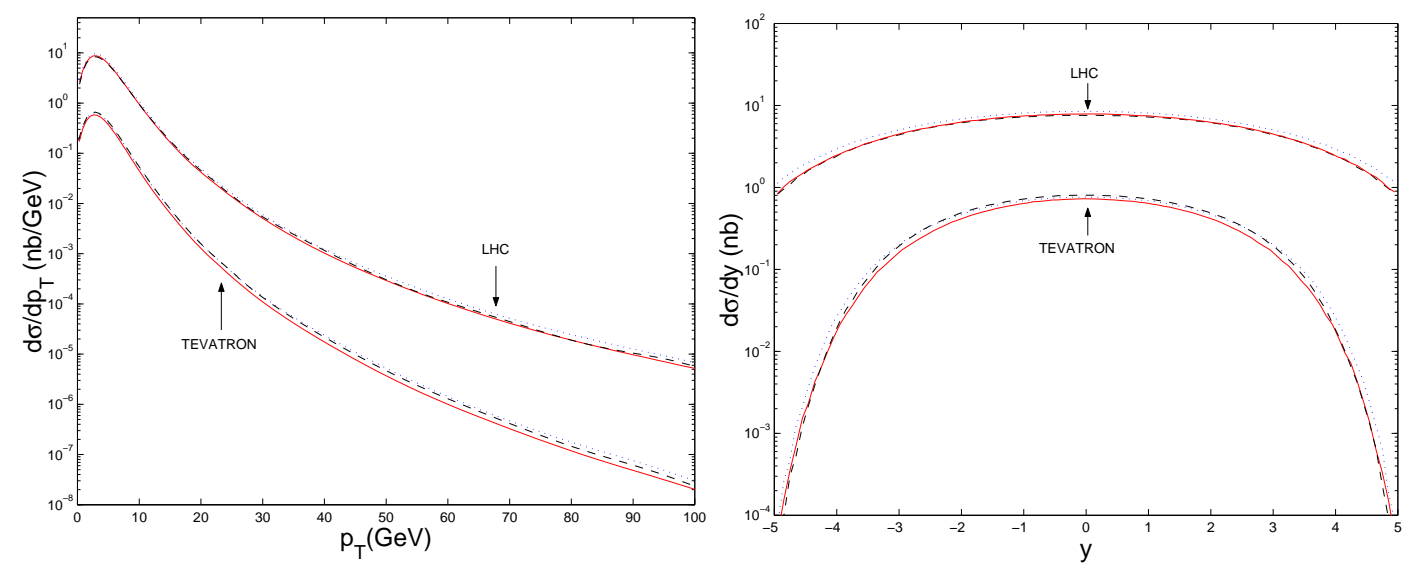

FIG. 2: $B_{c}$ differential distributions versus its transverse momentum $p_{T}$ and rapidity $y$ for different versions of gluon distributions in leading order. The characteristic energy scale is taken as $Q^{2}=\hat{s} / 4$. Solid line: CTEQ5L; dotted line: GRV98L and dashed line: MRST2001L. The upper (lower) three lines corresponding to the distributions in LHC (TEVATRON).

sections for TEVATRON and LHC, $R=\left(\frac{\sigma_{T E V A T R O N}}{\sigma_{L H C}}\right)_{p_{\text {Tcut }}}$ and put the results for $B_{c}\left[1^{1} S_{0}\right]$ in TABLE IV]

To show the uncertainties due to various factors more quantitatively, let us compute the differential cross sections of $p_{T}$ and $y$ for the pseudo-scalar $B_{c}$ meson and draw the curves accordingly.

From FIG. 2, we may see that the differential distributions for the three PDFs CTEQ5L, GRV98L and MRST2001L are very similar. The corresponding differences for TEVATRON and LHC are evident (the differences as shown in TABLE II] are less than 20\%). In regions of comparatively small $p_{T}$ and $y$ in TEVATRON the distributions of $p_{T}$ and $y$ obtained with MSRT2001L are the largest, and then those of GRV98L and CTEQ5L; while at LHC, those 

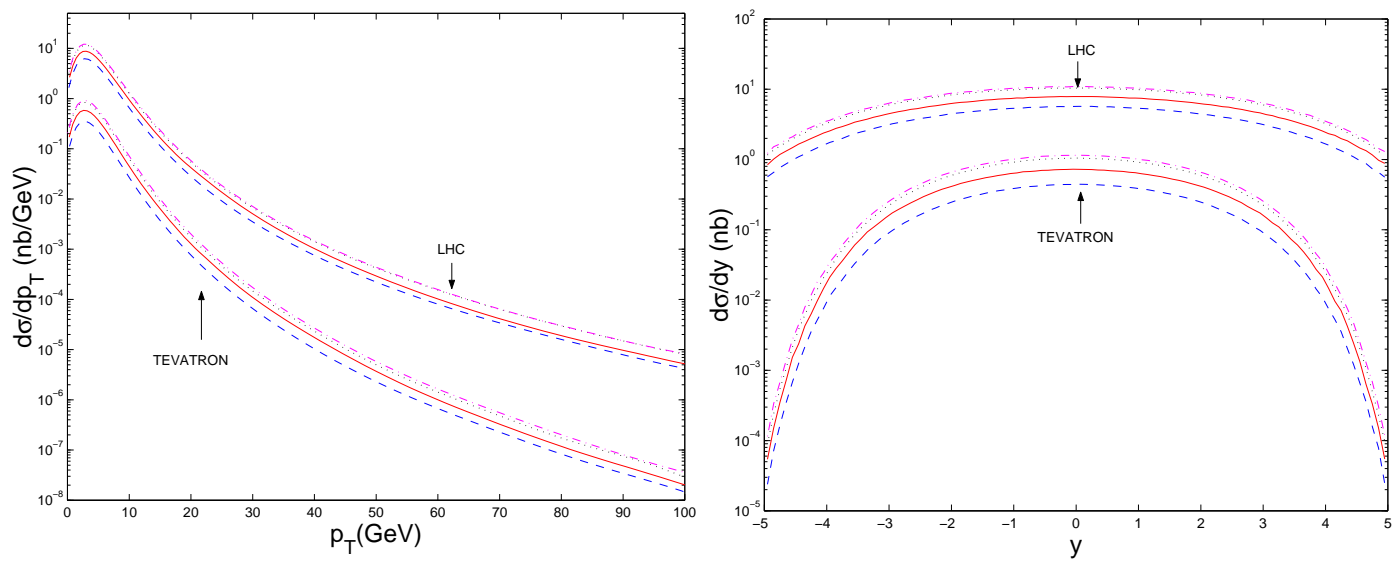

FIG. 3: $B_{c}$ differential distributions versus its transverse momentum $p_{T}$ and rapidity $y$ for four typical choices of the characteristic energy scale $Q^{2}$. The gluon distribution is chosen as CTEQ5L and the running $\alpha_{s}$ is in leading order. The choice of $Q^{2}$ is: solid line Type A; dotted line Type B; dashed line Type C and dash-dot line Type D. The upper (lower) four lines corresponding to the distributions at LHC (TEVATRON).

of GRV98L are the largest, and then those of CTEQ5L and MSRT2001L. From the figure, we also see that the $p_{T}$ distributions in TEVATRON are steeper than those in LHC.

Since in literature, various $\alpha_{s}$ have been adopted in making estimates of the $B_{c}$ production 8, 9, 10, 11, 12, 13] so to follow these authors, we try several $\alpha_{s}$ to compute the production. Namely, in addition to the LO $\alpha_{s}$, the constant $\alpha_{s}=0.22$ and the NLO $\alpha_{s}$ are also taken into the computations and the curves of the production obtained by the various $\alpha_{s}$ are drawn in FIG. 4

We may see from FIG. 4 that the variations of the results are quite large for both TEVATRON and LHC; those with $\alpha_{s}=0.22$ are the largest, those with NLO $\alpha_{s}$ are the smallest, while those with $\mathrm{LO} \alpha_{s}$ are in the middle.

In summary, of all the uncertainties which have been studied in this subsection, the uncertainty caused by the different choice of $Q^{2}$ is the largest, that can be as great as a factor around $\frac{1}{3}$ (although in the literature results with various choices of $\alpha_{s}$ running have given rise to even a greater change). 

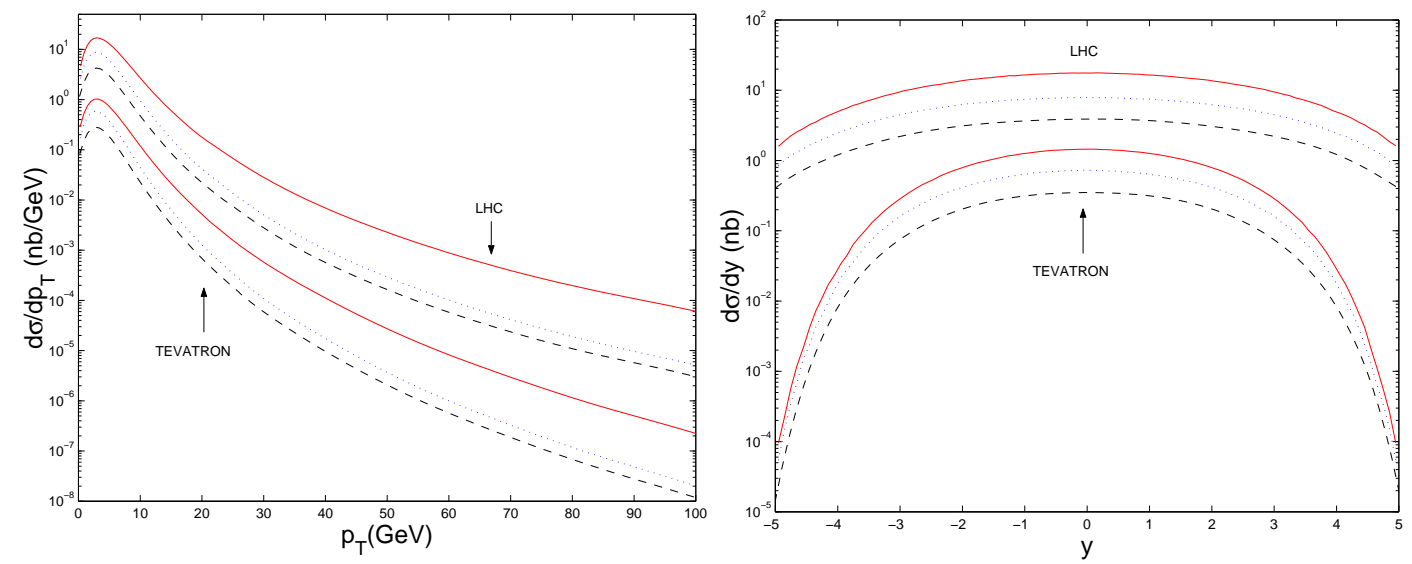

FIG. 4: $B_{c}$ differential distributions versus its transverse momentum $p_{T}$ and rapidity $y$ for different running $\alpha_{s}$. The gluon distribution of CTEQ5L and the Type A characteristic energy scale $Q^{2}=$ $\hat{s} / 4$, are used here. The solid line stands for the constant $\alpha_{s}=0.22$, the dotted line for the leading order running $\alpha_{s}$ and the dashed line for the next to leading order running $\alpha_{s}$. The upper (lower) four lines correspond to the distributions in LHC (TEVATRON).

\section{Comparison between the Mechanisms of Gluon-Gluon Fusion and Quark- Antiquark Annihilation}

In this subsection we make a brief comparison between the mechanisms of gluon-gluon fusion and quark-antiquark annihilation. Since, in a hadron such as $P, \bar{P}, \pi, \cdots$, the PDFs of heavy quarks are very small throughout the active region $\left|x_{i}\right| \leq 1$, it is sufficient to take into account only the light quark pair $(u-\bar{u}, d-\bar{d}$ and $s-\bar{s})$ annihilations.

The comparative results for the gluon-gluon fusion and the quark-antiquark annihilation mechanisms.In FIG 5, we show

From FIG 5 we see that for production at a high energy, such as TEVATRON and LHC, the gluon-gluon fusion mechanism is dominant over the quark-antiquark annihilation mechanism, typically when the transverse momentum $p_{T} \leq 60 \mathrm{GeV}$ and rapidity $|y| \leq$ 5.0. Only in TEVATRON do the contributions from gluon-gluon fusion become smaller than those from quark-antiquark annihilation when $p_{T} \geq 70 \mathrm{GeV}$. Generally speaking, for the transverse momentum distributions, at low $p_{T}$ the contributions from gluon-gluon fusion are greater than those from quark-antiquark annihilation by at least two orders of magnitude for both LHC and TEVATRON. For high transverse momentum $\left(p_{T} \geq 70 \mathrm{GeV}\right)$, 

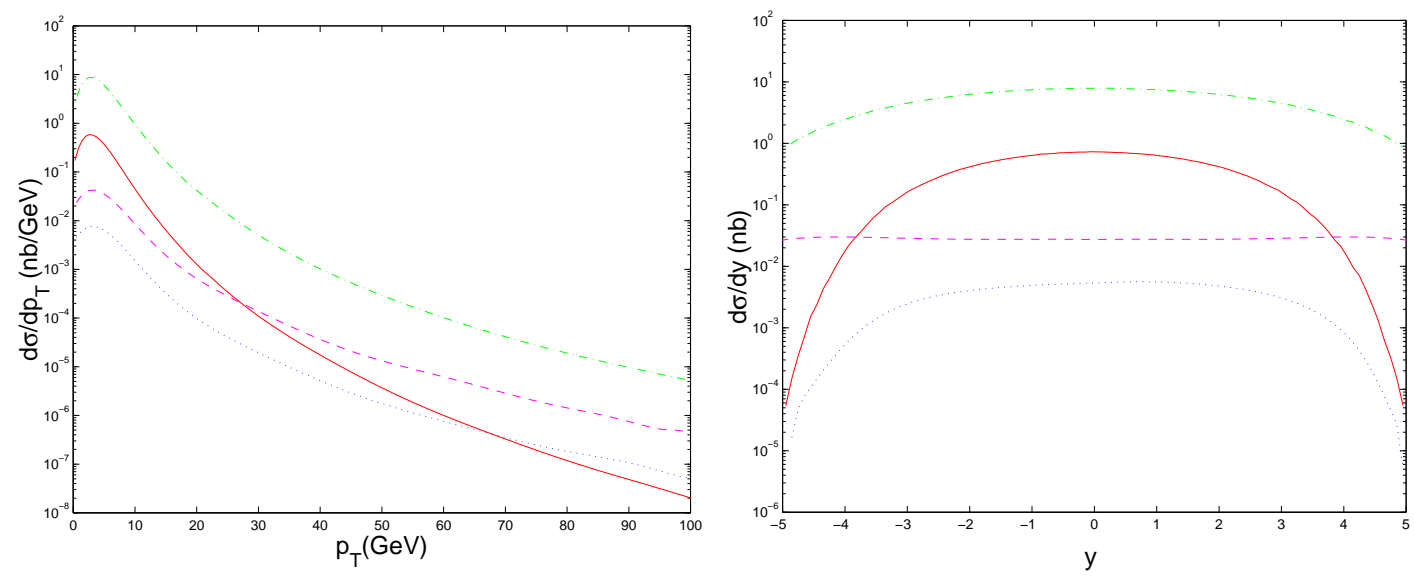

FIG. 5: $B_{c}$ distributions versus its transverse-momentum $p_{T}$ and rapidity $y$ of $B_{c}$ due to gluongluon fusion and quark-antiquark annihilation (the direction of positive $y$ is defined as that of the momentum of the colliding proton). The PDFs are taken from CTEQ5L, $\alpha_{s}$ running is set at leading order, and $Q^{2}=\frac{1}{4} \hat{s}$. The solid lines correspond to gluon-gluon fusion and the dotted lines to quark-antiquark annihilation in TEVATRON. The dashed-dotted lines correspond to gluon-gluon fusion and the dashed lines to quark-antiquark annihilation in LHC.

the difference in the two mechanisms changes sign in TEVATRON (i.e. contributions from quark-antiquark annihilation become greater than those from gluon-gluon fusion due to the fact that with such a high $p_{T}$, the valence quark PDFs play a significant role), but in LHC the contributions from gluon-gluon fusion are still greater by an order of magnitude than those from quark-antiquark annihilation. For rapidity $y$ distributions, in the $|y| \simeq 0$ region the contributions from gluon-gluon fusion are greater by two orders of magnitude in both colliders; but in TEVATRON in the higher region of $2.0 \leq|y| \leq 5.0$, contributions from the valance quarks' annihilations become more important, so that contributions from quark-antiquark annihilation increase remarkably (though still below those from gluon-gluon fusion). It is interesting to note that there is a slight asymmetry in the rapidity $y$ of the meson $B_{c}$ for the quark-antiquark annihilation subprocess. In TEVATRON it is of $P \bar{P}$ collision, so the asymmetry in $y$ is maintained somewhat (see FIG 5 ). We will describe the feature in TEVATRON elsewhere [30].

The contributions from quark-antiquark annihilation mechanism to $B_{c}$ hadronic production, compared with those from gluon-gluon fusion mechanism, can be ignored safely in LHC in the allowed kinematic region. In TEVATRON, they can also be ignored over most of the 
allowed kinematic region, i.e., only a little allowed kinematic region is an exception. In the paper, therefore, we consider the gluon-gluon fusion mechanism mainly.

\section{KINEMATIC CUTS FOR THE HADRONIC PRODUCTION OF $B_{c}$ MESON}

Experimentally, there is no detector which can cover all the kinematics of the events, so only some of $B_{c}$ produced events can be observed completely. For instance, in a high energy hadronic collider, the $B_{c}$ events with a small $p_{T}$ and/or a large rapidity $y$ (the produced $B_{c}$ mesons move very close to the beam direction) cannot be detected by the detectors at it directly, so this kind of events cannot be utilized for experimental studies in common cases. Therefore, only 'detectable' events should be taken into account in the estimates for a specific purpose, i.e., events with proper kinematic cuts on $p_{T}$ and $y$ must be put on precisely in the estimates. Considering detectors' abilities and to offer experimental references, we try various cuts accordingly in the estimate of the $B_{c}$ production.

First, we study the distributions of $p_{T}$ and $\sqrt{\hat{s}}$ for the $B_{c}$ meson with various rapidity cuts $y_{\text {cut }}$ (here we mean that only the $B_{c}$ events with $|y| \leq y_{\text {cut }}$ are taken into account), and as an example only the pseudo-scalar $B_{c}$ meson is taken into account. Note that the momenta and energies of the $b, \bar{c}$ and $B_{c}$ meson are measurable experimentally (a complete measurement of the events), hence the C.M. energy $\sqrt{\hat{s}}$ of the subprocess is measurable, i.e., in fact the distribution of $\sqrt{\hat{s}}$ is measurable experimentally. Take into account the abilities in measuring rapidity of $B_{c}$ for the detectors CDF, D0 and BTeV at TEVATRON, and ATLAS, CMS and LHC-B at LHC, various possible rapidity cuts, $y_{c u t} \sim 1.5$ or higher, are tried. To project out the cut effects, here we fix to use CTEQ5L for gluon distribution function, LO running $\alpha_{s}$ and Type A energy scale $\left(Q^{2}=\hat{s}^{2} / 4\right)$ to carry out the study. The results for the distributions of $p_{T}$ and $\hat{s}$ with various $y$-cuts are shown a similar behavior, thus we do not plot the curve here, but note that the dependence of the differential distributions on $y_{c u t}$ and $p_{T c u t}$ for LHC is stronger than that for TEVATRON. This is because that at TEVATRON, all the distributions with sizable rapidity cover a smaller region in $y$. The correlations between $p_{T}$ and $y$ are interesting, so we plot the $y$-distributions with various $p_{T^{-}}$cuts over a wide range $p_{\text {Tcut }}: 5.0 \sim 100 \mathrm{GeV}$ in FIG.6. From the figure (FIG.66), the dependence of the differential distributions on rapidity $y$ with different $p_{T c u t}$ at LHC exhibits a broader profile than that at TEVATRON. 

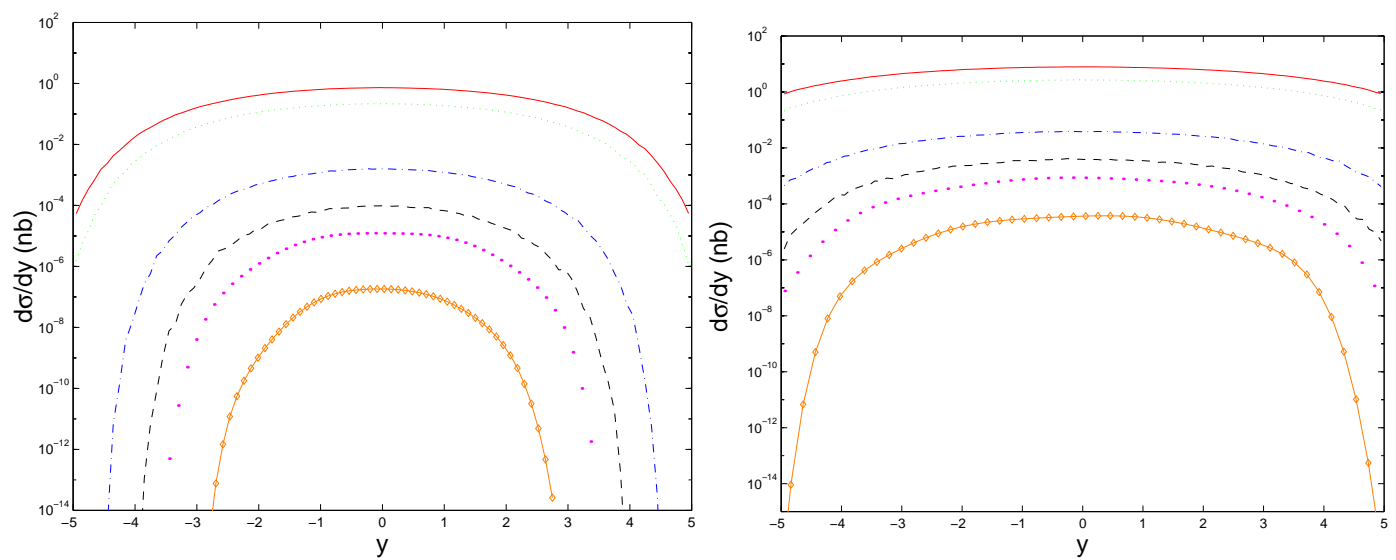

FIG. 6: $B_{c}$ differential distributions versus its $y$ with various $p_{T c u t}$ in TEVATRON (left diagram) and in LHC (right diagram). Solid line corresponds to the full production without $p_{\text {Tcut }}$; dashed line to $p_{\text {Tcut }}=5.0 \mathrm{GeV}$; dash-dot line to $p_{T \text { cut }}=20.0 \mathrm{GeV}$; the dashed line to $p_{\text {Tcut }}=35.0 \mathrm{GeV}$; the big dotted line to $P_{\text {Tcut }}=50.0 \mathrm{GeV}$ and the solid line with diamonds to $p_{\text {Tcut }}=100 \mathrm{GeV}$.

The $p_{T}$-distributions of the production vary with $y_{c u t}$ mainly due to the fact that as $p_{T}$ increases, the dependence of the distribution on $y$ becomes smaller as the value of $y_{\text {cut }}$ becomes less important. When $p_{T}$ increases to sufficient large value, the 'border' of the differential distributions (see FIG. 6) becomes smaller than $y_{\text {cut }}$ and the $p_{T}$ differential distributions with and without $y$-cut is coincide.

To analyze the quantitative difference of the differential distributions with regard to $p_{T c u t}$ and $y_{c u t}$, we introduce a ratio for the integrated hadronic cross sections:

$$
R_{p_{\text {Tcut }}}=\left(\frac{\sigma_{y_{c u t}}}{\sigma_{0}}\right)_{p_{\text {Tcut }}}
$$

where $\sigma_{y_{c u t}}$ and $\sigma_{0}$ are the hadronic cross section with and without $y_{\text {cut }}$ respectively. The ratio $R_{p_{T c u t}}$ varies with $p_{T c u t}$ and $y_{c u t}$, and its values are given in TABLE $\nabla$

From TABLE $\nabla$ we may see that, for a fixed $y_{c u t}$, the value of $R_{p_{T c u t}}$ becomes larger with increasing $p_{\text {Tcut }}$. It is understandable that the differential distributions versus the rapidity $y$ decrease with the increment of $p_{T}$, so the contributions to the hadronic cross section surviving after the cut, i.e. $\left(|y| \leq y_{\text {cut }}\right)$, increase with the increment of $p_{T c u t}$.

As for the $B_{c}$ meson production, only in high energy hadronic colliders can numerous $B_{c}$ mesons be produced. The cross section of $B_{c}$ meson production is quite sensitive to the C.M. energy of the collider. Since RUN-II of TEVATRON is running now at a slightly higher energy than that $1.8 \mathrm{TeV}$ (of RUN-I), we take TEVATRON as an example to study 
TABLE V: Values of the ratio $R_{p_{T c u t}}$ (see definition in text) for the hadronic production of pseudoscalar $B_{c}$ meson in TEVATRON and LHC.

\begin{tabular}{|c||c|c|c||c|c|c||c|c|c||c|c|c||c|c|c|}
\hline \hline \multicolumn{1}{|c||}{$p_{\text {Tcut }}$} & \multicolumn{2}{|c||}{$0.0 \mathrm{GeV}$} & \multicolumn{3}{c||}{$5 \mathrm{GeV}$} & \multicolumn{3}{c||}{$20 \mathrm{GeV}$} & \multicolumn{3}{|c||}{$35 \mathrm{GeV}$} & \multicolumn{3}{|c|}{$50 \mathrm{GeV}$} \\
\hline$y_{\text {cut }}$ & 1.0 & 1.5 & 2.0 & 1.0 & 1.5 & 2.0 & 1.0 & 1.5 & 2.0 & 1.0 & 1.5 & 2.0 & 1.0 & 1.5 & 2.0 \\
\hline \hline$R_{p_{\text {Tcut }}}($ TEVATRON) & 0.45 & 0.64 & 0.79 & 0.46 & 0.65 & 0.80 & 0.57 & 0.77 & 0.91 & 0.65 & 0.85 & 0.95 & 0.70 & 0.90 & 0.98 \\
\hline$R_{p_{\text {Tcut }}}(\mathrm{LHC})$ & 0.31 & 0.46 & 0.59 & 0.32 & 0.47 & 0.60 & 0.38 & 0.54 & 0.69 & 0.42 & 0.60 & 0.74 & 0.45 & 0.64 & 0.79 \\
\hline \hline
\end{tabular}

TABLE VI: The integrated hadronic cross section for TEVATRON at different C.M. energies. The gluon distribution is chosen from CTEQ5L and the characteristic energy scale of the production is chosen as Type A, i.e. $Q^{2}=\hat{s} / 4$. In addition, a cut for transverse momentum $p_{T}\left(p_{T}<5 \mathrm{GeV}\right)$ and a cut for rapidity $y(|y|>1.5)$ have been imposed.

\begin{tabular}{|c||c|c|c|c|}
\hline \hline C.M. energy & $1.8(\mathrm{TeV})$ & $1.9(\mathrm{TeV})$ & $1.96(\mathrm{TeV})$ & $2.0(\mathrm{TeV})$ \\
\hline \hline$B_{c}\left[{ }^{1} S_{0}\right]$ & 0.40 & 0.44 & 0.46 & 0.47 \\
\hline$B_{c}^{*}\left[{ }^{3} S_{1}\right]$ & 1.00 & 1.09 & 1.14 & 1.18 \\
\hline \hline
\end{tabular}

the increase caused by slight changes in the C.M. energy. The C.M. energy for RUN-II is designed to be $2.0 \mathrm{TeV}$, so here we take energy values, 1.8, 1.9, 1.96 and $2.0 \mathrm{TeV}$ to compute the cross sections. The obtained results for the values of the integrated cross-section with suitable cuts are shown in TABLE VI] From TABLE VI, we see that when the C.M. energy of TEVATRON increases from 1.8 to $2.0 \mathrm{TeV}$, the cross-section increases by about $20 \%$.

\section{SUMMARY}

In this paper we have presented quantitative studies on the uncertainties in estimates of the $B_{c}$ meson hadronic production. The uncertainties have been examined in turn by 'factorizing' their origins. The computations are based on the generator BCVEGPY 23] mainly. The investigated quantitatively uncertainties involve those due to various versions of PDFs 
given by various groups, the variations of the parameters relevant to the potential model, the strong coupling $\alpha_{s}$ relevant to its running and the characteristic energy scale $Q^{2}$ of the process where the QCD factorization is carried out and etc.. We find that the characteristic energy scale $Q^{2}$ of the process is the source which causes the greatest uncertainty for the LO QCD estimates. We have also shown the differences between LHC and TEVATRON for various observables with reasonable kinematic cuts, such as the cuts on the $B_{c}$ meson transverse momentum $p_{\text {Tcut }}$ and rapidity $y_{c u t}$. We also point out that at TEVATRON from RUN-I to RUN-II due to the increase of the collision C.M. energy, the cross-section of the $B_{c}$ production increase by about $20 \%$. In view of the fact that TEVATRON is running while LHC is still under constructing, based on our studies we can clearly see that experimental $B_{c}$ studies at the two colliders are stimulative and complimentary. As for the topic to study of $B_{s}-\bar{B}_{s}$ mixing and $C P$ violations in $B_{s}$ meson decays at high energy hadronic colliders with tagged $B_{s}$ mesons produced through the $B_{c}$ decays, since LHC has much higher C.M. energy, so the $B_{c}$-production cross-section is higher than one order of magnitude at TEVATRON, and LHC has much higher luminosity than at TEVATRON, it seems that the particularly interesting above topic may be more accessible and fruitful at LHC than that at TEVATRON RUN-II, even RUN-III.

Acknowledgement The authors would like to thank Yu-Qi Chen and Guo-Ming Chen for discussions, and to thank Vaia Papadimitriou for the suggestion to compute the production at various C.M. energies for TEVATRON. This work was supported in part by Nature Science Foundation of China (NSFC).

[1] DELPHI Collaboration, P. Abreu et al. Phys. Lett. B 398, 207 (1997); ALEPH Collaboration, R. Barate et al. Phys. Lett. B 402, 213 (1997); OPAL Collaboration, K. Ackerstaff et al. Phys. Lett. B 420, 157 (1998).

[2] CDF Collaboraten, F. Abe, et al., Phys. Rev. Lett. 77, 5176 (1996).

[3] CDF Collaboraten, F. Abe, et al., Phys. Rev. Lett. 81, 2432 (1998); Phys. Rev. D58, 112004 
(1998).

[4] K. Anikeev, et al., hep-ph/0201071.

[5] Chao-Hsi Chang, Proceedings of the XXXVIIth RENCONITRES DE MORIOND Les Arcs, Savoie, France, 2002 QCD and High Energy Hadronic Interactions, p-27, hep-ph/20205112.

[6] C. Quigg, Proceedings of the Workshop on B Physics at Hadron Accelerators, Snowmass (CO) USA, 1993, Eds. P. McBride and C.S. Mishra.

[7] Chao-Hsi Chang and Yu-Qi Chen, Phys. Rev. D 46, 3854 (1992); Erratum Phys. Rev. 50, 6013 (1994).

[8] Chao-Hsi Chang and Yu-Qi Chen, Phys. Rev. D 48, 4086 (1993).

[9] E.Braaten, K. Cheung and T.C. Yuan, Phys. Rev. D 48, 4230 (1993); E. Braaten, K. Cheung and T.C. Yuan, Phys. Rev. D 48, R5049 (1993).

[10] Chao-Hsi Chang, Yu-Qi Chen, Guo-Ping Han and Hung-Tao Jiang, Phys. Lett. B 364, 78 (1995); Chao-Hsi Chang, Yu-Qi Chen and R. J. Oakes, Phys. Rev. D 54, 4344 (1996); K. Kolodziej, A. Leike and R. Rückl, Phys. Lett. B 355, 337 (1995).

[11] A.V. Berezhnoy, V.V. Kiselev, A.K. Likhoded, Z. Phys. A 356, 79 (1996); S.P. Baranov, Phys. Rev. D 56 3046, (1997).

[12] K. Cheung, Phys. Lett. B 472, 408 (2000).

[13] A.V. Berezhnoy, V.V. Kiselev, A.K. Likhoded and A.I. Onishchenko, Phys. Atom. Nucl. 60, 1729 (1997); hep-ph/9703341.

[14] Yu-Qi Chen and Yu-Ping Kuang, Phys. Rev. D 46, 1165 (1992); E. Eichten, C. Quigg, Phys. Rev. D 495845 (1994); Phys. Rev. D 52, 1726 (1995); S.S. Gershtein, V.V. Kiselev, A.K. Likhoded and A.V. Tkabladze, Phys. Rev. D 51, 3613 (1995).

[15] C. T. H. Davies, et al., Phys. Lett. B 382, 131 (1996).

[16] Chao-Hsi Chang, Yu-Qi Chen, Phys. Rev. D49, 3399 (1994); Chao-Hsi Chang and Yu-Qi Chen, Commun. Theor. Phys. 23 (1995) 451.

[17] A. Abd El-Hady, J.H. Munoz and J.P. Vary; Phys. Rev. D62 014014 (2000).

[18] N. Isgur, D. Scora, B. Grinstein and M. Wise, Phys. Rev. D 39, 799 (1989); M. Lusignoli and M. Masetti, Z. Phys. C 51, 549 (1991); D. Scora and N. Isgur, Phys. Rev. D 52, 2783 (1995); Dongsheng Du, G.-R. Lu and Y.-D. Yang, Phys. Lett. B 387, 187 (1996); Dongsheng Du, et al., Phys. Lett. B 414, 130(1997); Jia-Fu Liu and Kuang-Ta Chao, Phys. Rev. D 564133 , 
(1997); P. Colangelo and F.De Fazio, Phys. Rev. D 61034012 (2000); V.V. Kiselev, A.E. Kovalsky and A.K. Likhoded, Nucl. Phys. B 585353 (2000); V.V. Kiselev, A.K. Likhoded and A.I. Onishchenko, Nucl. Phys. B 569 473, (2000); M.A. Nobes and R.M. Woloshyn, J. Phys. G 26 1079, (2001).

[19] M.A. Ivanov, J.G. Körner and O.N. Pakhomova, Phys. Lett. B 555, 189 (2003); A. Faessler et al, Eur. Phys. J. direct C 4, 18 (2002); M.A. Ivanov, J.G. Körner and P. Santorelli, Phys. Rev. D 63, 074010 (2001).

[20] Chao-Hsi Chang, Shao-Long Chen, Tai-Fu Feng and Xue-Qian Li, Phys. Rev. D 64, 014003 (2001); Commun. Theor. Phys. 35, 51 (2001).

[21] M. Beneke and G. Buchalla, Phys. Rev. D 53, 4991 (1996).

[22] Chao-Hsi Chang, J.-P. Cheng and C.-D. Lü, Phys. Lett. B 425, 166 (1998); P. Colangelo and F. De Fazio, Mod. Phys. Lett. A 14, 2303 (1999); Chao-Hsi Chang, Cai-Dian Lü, GuoLi Wang and Hong-Shi Zong, Phys. Rev. D60 114013, 1999; Chao-Hsi Chang, Yu-Qi Chen, Guo-Li Wang and Hong-Shi Zong, Phys. Rev. D 65, 014017 (2001); Commun. Theor. Phys. 35, 395 (2001); Chao-Hsi Chang, Anjan K. Giri, Rukmani Mohanta and Guo-Li Wang, J. Phys. G 28, 1403, (2002), hep-ph/0204279 Xing-Gang Wu, Chao-Hsi Chang, Yu-Qi Chen and Zheng-Yun Fang, Phys. Rev. D, 67, 094001 (2003), hep-ph/0209125.

[23] Chao-Hsi Chang, Chafik Driouich, Paula Eerola and Xing-Gang Wu, Comput.Phys.Commun. 159, 192(2004); hep-ph/0309120.

[24] T. Sjostrand, Comput. Phys. Commun. 82 (1994) 74.

[25] Geoffrey T. Bodwin, Eric Braaten and G. Peter Lepage, Phys. Rev. D, 51, 1125 (1995); Erratum Phys. Rev. D, 55, 5853 (1997).

[26] H.L. Lai, et al., Eur. Phys. J.C12, 375(2000).

[27] H.L. Lai, et al., hep-ph/0201195

[28] M. Glueck, E. Reya, A. Vogt, Eur. Phys. J.C5, 461(1998).

[29] A.D. Martin, R.G. Roberts, W.J. Stirling and R.S. Thorne, Eur. Phys. J. C23, 73(2002).

[30] Chao-Hsi Chang and Xing-Gang Wu, The mechanism of quark-antiquark annihilation in hadronic production of the meson $B_{c}$, in preparation.

[31] In PYTHIA the value of $Q^{2}$ can be definitely fixed at each interaction vertex, therefore when applying PYTHIA to the production, there is no energy scale ambiguity. However, in PYTHIA all kinds of $b$-hadron $\left(B_{s}, B_{c}, \Lambda_{b}, \cdots\right)$ events will be produced according to the proper fragmen- 
tation possibilities, among which the fragmentation possibility for $B_{c}$ is quite small [7, 9], thus in terms of PYTHIA to generate the $B_{c}$ events is not 'economical', i.e., when simulating the $B_{c}$ events, enormous unwanted $b$-hadron events with higher probability will be generated in the meantime. By contrast, the approach which we are adopted here has quite high efficiency to generate the $B_{c}$ events, though the uncertainties from the choices of $Q^{2}$ are unavoidable. 